Article

\title{
The Effect of Ageing on Chemical and Mechanical Properties of Asphalt Mortar
}

\author{
Ruxin Jing ${ }^{1, *} \mathbb{C}$, Xueyan Liu ${ }^{1}$, Aikaterini Varveri ${ }^{1}{ }^{\circledR}$, Athanasios Scarpas ${ }^{1,2}$ and \\ Sandra Erkens ${ }^{1}$ (D) \\ 1 Pavement Engineering, Delft University of Technology, 2628 CN Delft, The Netherlands; \\ x.liu@tudelft.nl (X.L.); a.varveri@tudelft.nl (A.V.); a.scarpas@tudelft.nl (A.S.); s.m.j.g.erkens@tudelft.nl (S.E.) \\ 2 Civil Infrastructure and Environmental Engineering Department, Khalifa University, Abu Dhabi 127788, UAE \\ * Correspondence: r.jing@tudelft.nl; Tel.: +31-061-879-9589
}

Received: 8 October 2018; Accepted: 9 November 2018; Published: 13 November 2018

check for updates

\begin{abstract}
Asphalt mortar is a mixture of bitumen, filler, and sand. Mortar plays an important role in asphalt mixtures as it serves as the adhesive between the coarser aggregates. Due to the effect of bitumen ageing, the chemical and mechanical properties of asphalt mortar evolve with time. The mortar becomes more brittle and prone to cracking, thus leading to inferior pavement performance. In this study, Fourier transform infrared (FTIR) spectrometry was used to quantify changes in the chemical functional groups related to ageing and to calculate the carbonyl and sulfoxide indices. In addition, frequency sweep tests and uniaxial tension tests were performed by means of dynamic shear rheometer (DSR) tests to determine evolution of the stiffness and strength due to ageing. Two different oven ageing protocols were used to evaluate the effect of fine mineral particles on bitumen ageing. The protocols differed with respect to the order of ageing and mixing of the constituents. The results showed that both the chemical and mechanical properties of mortars significantly changed with ageing. Specifically, the carbonyl index, stiffness, and strength of the mortar increased. Under the same ageing conditions, a higher ageing level was observed for mortars produced by first mixing and then ageing compared to the mortars produced by mixing aged bitumen with filler and sand. This could be due to the presence of sand and filler particles, which resulted in an increased length of diffusion paths and consequently a slower ageing process.
\end{abstract}

Keywords: asphalt mortar; ageing; FTIR; stiffness; strength

\section{Introduction}

Ageing of bitumen is believed to be a major contributor to many pavement distresses, such as raveling, cracking, and fatigue [1-6]. Ageing occurs during the process of storage, mixing, transportation, construction, and service. Studies have demonstrated that ductility and penetration of bitumen diminish as a result of ageing, while softening point and ignition temperature increase in the process [7-9]. Moreover, the viscosity increases and bitumen becomes stiffer.

Asphalt mortar, commonly known as fine aggregate matrix, is a mixture of bitumen, filler, and sand. It is the binding medium that holds the aggregates of an asphalt mixture together, and it plays an important role as the link between bitumen properties and mixture performance. Past studies have shown that the testing of bituminous mortar is an efficient and repeatable approach to predict the performance of asphalt mixture [10-12]. The ageing of bitumen, through the asphalt mortar as an intermediate medium, causes the mixture to become excessively hard and brittle and consequently more susceptible to producing pavement distress [13]. For this reason, some studies have considered asphalt mortar ageing. For instance, Cui et al. investigated the effect of laboratory long-term ageing on the rheological properties of asphalt mortar. The long-term ageing test is a combination of 
ultraviolet ageing, thermal ageing, and humidity [14]. Molenaar et al. studied the effects of ageing on the mechanical characteristics of bituminous binders in porous asphalt (PA) concrete. The results showed that ageing increased the tensile strength of the bituminous binders but decreased the strain at break [15]. In addition, the fatigue results showed that the adhesive zone between stone and mortar tended to be the weakest link because there was a bitumen-rich interlayer [16]. Tong et al. evaluated the moisture and ageing damage of asphalt mortar using the repeated direct tensional test method. The results showed that fatigue crack growth of asphalt mortar increased substantially after ageing [17]. Zhang et al. quantified the ageing effect on the rheological characteristics of two types of asphalt mortar and applied the results to evaluate the raveling resistance of PA wearing courses in finite element simulations. It was found that ageing had more influence on the complex shear modulus of the base mortar compared to the SBS (Styrene-Butadiene-Styrene) mortar, but its effects on fatigue resistance were the opposite [18]. These studies proved that mortar ageing had a negative influence on pavement performance, such as fatigue and raveling. However, the studies were limited in considering the rheological changes of asphalt mortar subject to various conditions.

The main objective of this study was to investigate the effect of filler and sand on ageing and determine the evolution of mortar properties due to ageing. To achieve this, two different ageing conditions were applied on mortar by an oven. The ageing method differed with respect to the order of ageing and mixing of the constituents. The chemical composition of asphalt mortar was evaluated by means of Fourier transform infrared (FTIR) spectrometer. Cylindrical mortar samples were then prepared and tested using dynamic shear rheometer (DSR) tests. In addition, the original bitumen was tested after the same ageing conditions as that of asphalt mortar.

\section{Materials and Methods}

\subsection{Materials and Asphalt Mortar Mix Design}

The PEN 70/100 bitumen, which is one of the most commonly used bitumen in the Netherlands, was used in this study. Table 1 shows the main physical and rheological properties of the PEN $70 / 100$ bitumen. The mortars were prepared using river sand, which is a type of sand with quartz, with a nominal maximum size of $2 \mathrm{~mm}$ and Wigro $60 \mathrm{~K}$ filler, which is a type of filler with hydroxide that contains $25 \%$ hydrated lime (density is $2780 \mathrm{~kg} / \mathrm{cm}^{3}$ ).

Table 1. Specifications of PEN 70/100 at fresh (unaged) state.

\begin{tabular}{ccc}
\hline Property & Unit & PEN 70/100 \\
\hline Penetration at $25^{\circ} \mathrm{C}$ & $0.1 \mathrm{~mm}$ & $70-100$ \\
Softening point & ${ }^{\circ} \mathrm{C}$ & $43-51$ \\
Dynamic viscosity at $60^{\circ} \mathrm{C}$ & $\mathrm{Pa} \mathrm{s}$ & 160 \\
Complex shear modulus at $1.6 \mathrm{~Hz}$ and $60^{\circ} \mathrm{C}$ & $\mathrm{kPa}$ & 1.8 \\
Phase angle at $1.6 \mathrm{~Hz}$ and $60^{\circ} \mathrm{C}$ & $\circ$ & 88 \\
\hline
\end{tabular}

The mortar was designed based on the mix design of two types of asphalt mixtures-porous asphalt (PA) 16 and stone mastic asphalt (SMA) 16-which represent an open and a dense-graded asphalt mixture, respectively, and are widely used in the Netherlands. Both PA 16 and SMA 16 consist of crushed stones with a nominal maximum aggregate size of $16 \mathrm{~mm}$, river sand, Wigro 60K filler, and PEN 70/100 bitumen. The binder contents were 5.0\% and 6.4\% for the PA and the SMA mixture, respectively. The aggregate gradations of PA16 and SMA 16 mixtures are shown in Figure 1. 


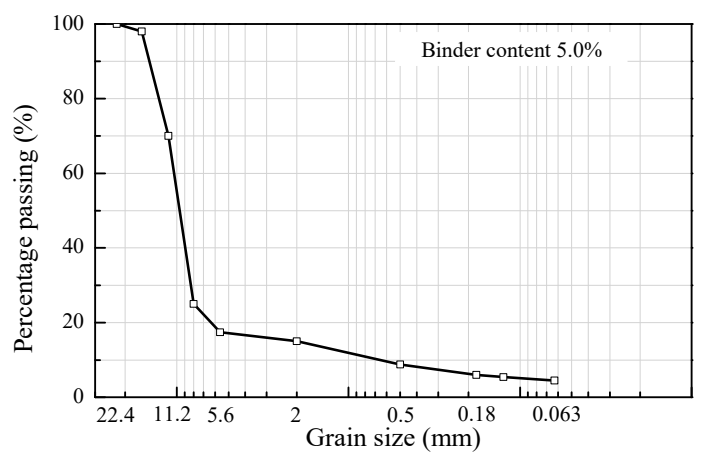

(a)

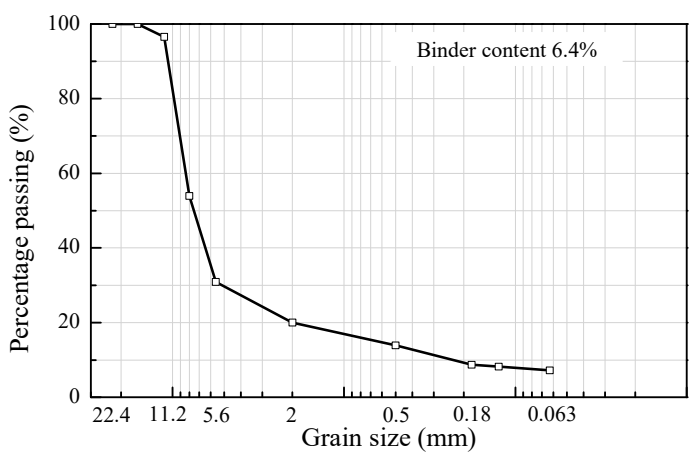

(b)

Figure 1. Aggregate gradation and physical properties of mixture: (a) porous asphalt (PA) 16; (b) stone mastic asphalt (SMA) 16.

The asphalt mortar used in this study contained sand with size smaller than $2 \mathrm{~mm}$. On the basis of gradations of PA 16 and SMA 16 in Figure 1, the mass ratios of bitumen, filler, and sand of each mixture were calculated and are presented in Table 2. Specifically, it was 1:0.9:2.1 for the mortar in PA 16 and 1:1.25:2 for the mortar in SMA 16. Therefore, considering an average between the mortars in PA 16 and SMA 16, the mass ratio of each composition in the designed mortar was selected as 1:1:2. This mortar composition was employed in the lab investigation.

Table 2. Asphalt mortar recipe.

\begin{tabular}{cccc}
\hline Mix Design & Bitumen & Filler & Sand \\
\hline Mortar in PA 16 & 1 & 0.9 & 2.1 \\
Mortar in SMA 16 & 1 & 1.125 & 2 \\
Designed Mortar & 1 & 1 & 2 \\
\hline
\end{tabular}

\subsection{Ageing Program}

First, the mortar samples were tested without applying any ageing protocol so as to define their properties at unaged (fresh) conditions. Then, two oven ageing protocols that were different in the order of ageing and mixing of the constituents were applied. Specifically, in the first method, the mortar constituents-bitumen, filler, and sand-were mixed after they were aged separately in the oven (denoted as AM); this method is commonly used as laboratory ageing protocol. In contrast, the second method involved first mixing the constituents to prepare the mortar and then subjecting the mortar samples to ageing (denoted as MA); this method is close to the ageing condition in the field. The purpose of having two different protocols was to identify the effect of filler and sand on ageing. Table 3 summarizes the ageing protocols that were considered. Specifically, the temperature was selected as $100{ }^{\circ} \mathrm{C}$ for all ageing tests, and the time varied from 20 to $160 \mathrm{~h}$. In addition, bitumen samples after the application of various ageing times were studied.

Table 3. Ageing program.

\begin{tabular}{ccccc}
\hline Ageing Temperature $\left({ }^{\circ} \mathbf{C}\right)$ & Ageing Time (h) & Mortar_AM & Mortar_MA & Bitumen \\
\hline \multirow{2}{*}{100} & 20 & $\sqrt{ }$ & & $\sqrt{ }$ \\
& 40 & $\sqrt{ }$ & $\sqrt{ }$ & $\sqrt{ }$ \\
& 80 & $\sqrt{ }$ & $\sqrt{ }$ & $\sqrt{ }$ \\
\hline
\end{tabular}

\subsection{Sample Preparation}

As the studied mortar samples were composed using fractions of aggregates up to $2 \mathrm{~mm}$, the parallel-plates configuration could no longer be applied. Instead of the parallel-plates configuration, 
a special set-up with clamps for cylindrical mortar sample was used for the DSR tests $[19,20]$ (Figure 2a). The preparation procedure of the mortar samples was adopted from a previous study at TU Delft [21]. Initially, the PEN 70/100 bitumen, filler, and sand were separately heated to $150{ }^{\circ} \mathrm{C}$. Then, the three compounds were mixed manually for 10-15 min. After the mixing process, asphalt mortar was poured into a silicone mold (Figure 2b). Two metal rings were prefixed on the top and bottom side of the mold. These metal rings ensured load transfer from the DSR into the samples (Figure 2c). After filling the mold with mortar, the mold was placed in the oven at $150{ }^{\circ} \mathrm{C}$ for $5 \mathrm{~min}$ to allow the suppression of possible air pockets that were formulated during pouring of the mix into the mold. Afterwards, the mold was cooled down to ambient temperature and then placed in a refrigerator at $-15^{\circ} \mathrm{C}$ for at least $2 \mathrm{~h}$. Finally, the mortar samples were removed from the mold and checked visually for air voids at the surface of the sample. The accepted samples were placed in a special silicone mold and stored in the refrigerator at $-15^{\circ} \mathrm{C}$ before testing (Figure $2 \mathrm{~d}$ ).

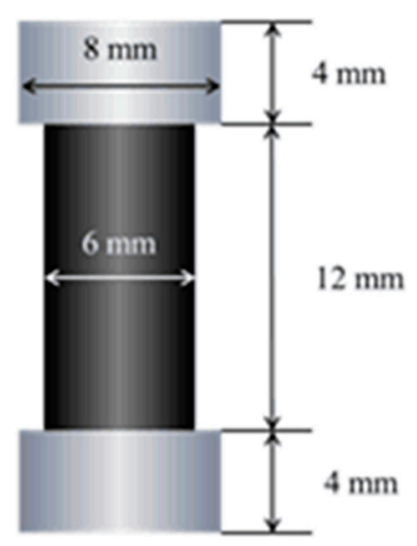

(a)

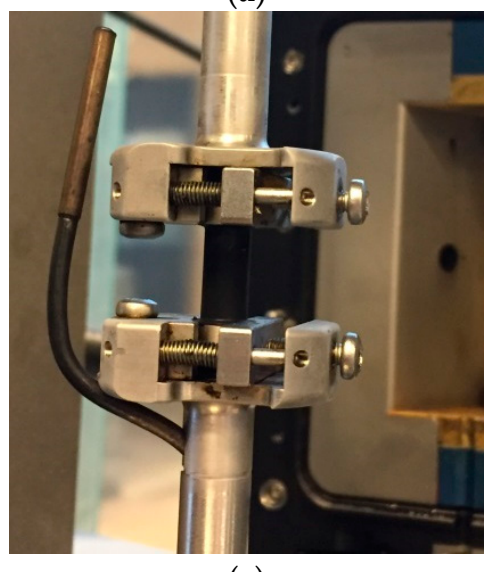

(c)

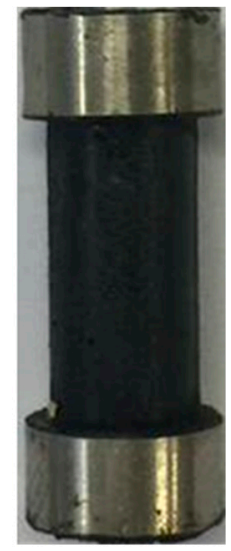

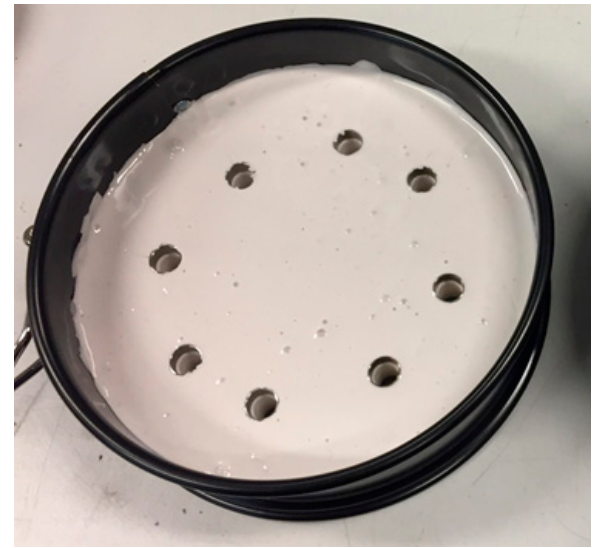

(b)

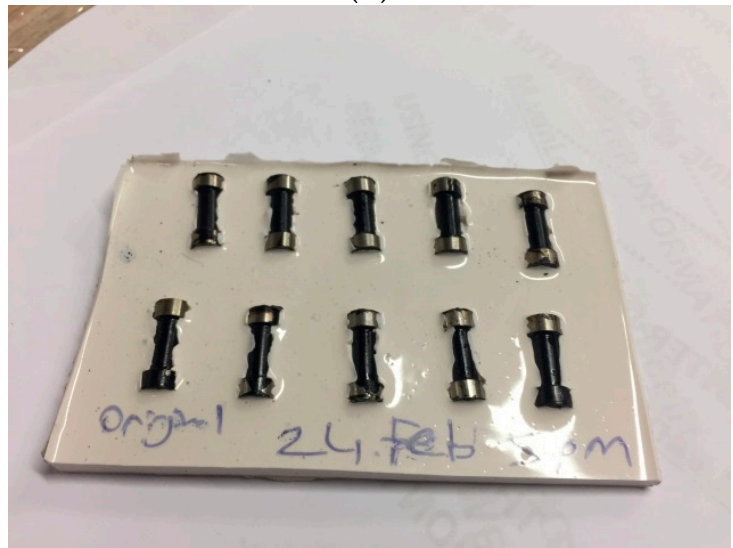

(d)

Figure 2. Asphalt mortar sample: (a) geometry of sample; (b) silicone mold for sample preparation; (c) measuring system for cylinder sample; (d) silicone mold for sample storage.

\subsection{Experimental Methods}

As an efficient, convenient, and inexpensive tool, the FTIR is widely used to determine the chemical characteristics of bituminous materials. In the infrared spectrogram of bitumen, the absorbance bands corresponding to carbonyls and sulfoxides show a significant increase with ageing [22-24]. The chemical characterization in this study was performed using the Spectrum 100 FT-IR spectrometer from PerkinElmer. A single-beam configuration was used. The sample was scanned 20 times with a fixed instrument resolution of $4 \mathrm{~cm}^{-1}$. The wavenumbers were set to vary from 600 to $4000 \mathrm{~cm}^{-1}$. At least three repetition tests were performed for each ageing condition. 
DSR is commonly used to investigate the rheological properties of bituminous materials [25-27]. In this study, the linear viscoelastic (LVE) strain range of the bitumen and asphalt mortar samples were initially determined using amplitude sweep tests. Within the LVE strain range, frequency sweep tests were performed at four different temperatures $\left(0,10,20\right.$, and $\left.30^{\circ} \mathrm{C}\right)$. During the tests, the frequency varied in a logarithmic manner from $50 \mathrm{~Hz}$ to $0.01 \mathrm{~Hz}$. Uniaxial tensile tests were performed at $0{ }^{\circ} \mathrm{C}$ with $0.2 \%(0.024 \mathrm{~mm} / \mathrm{s})$ tensile rate. The load and deformation in the testing process were automatically collected with a frequency of two measurements per second.

\section{Results and Discussion}

\subsection{Fourier Transform Infrared Spectrometer}

\subsubsection{FTIR Spectra}

The FTIR spectra were obtained in a wavenumber range from 4000 to $600 \mathrm{~cm}^{-1}$. However, the changes caused by ageing could be found at wavenumbers lower than $2000 \mathrm{~cm}^{-1}$, as shown in Figure 3. These wavenumbers corresponded to functional groups related to the oxidation processes. It showed that the carbonyl (left, red part) and sulfoxide functional (right, blue part) groups changed considerably after laboratory ageing, i.e., they both increased with ageing.

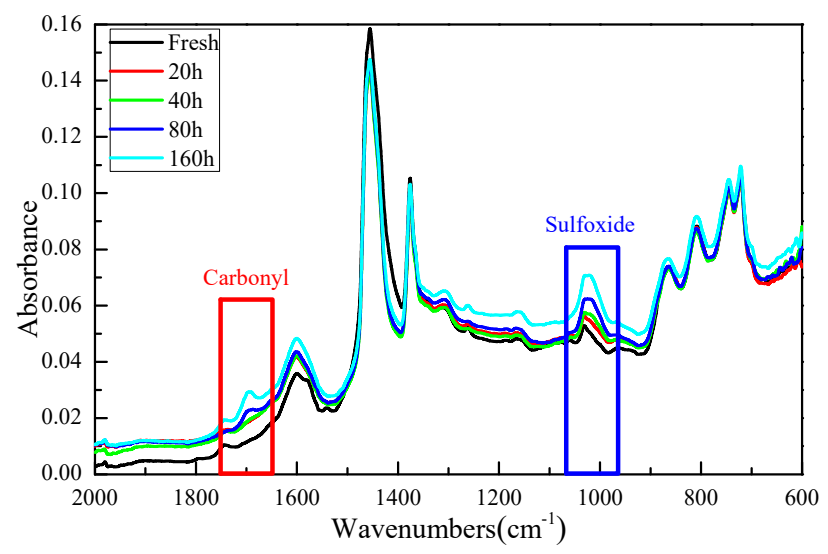

Figure 3. FTIR spectra of PEN 70/100 at different ageing hours.

The FTIR spectra of fresh mortar sample and each component (fresh bitumen, filler, and sand) are shown in Figure 4. It can be observed that the mortar-a mixture of bitumen, filler, and sand - had all functional groups as its three component materials. Figure 4 clearly shows that the carbonyl group that was present in the mortar originated only from the presence of bitumen, whereas sulfoxides can be present in all three components.

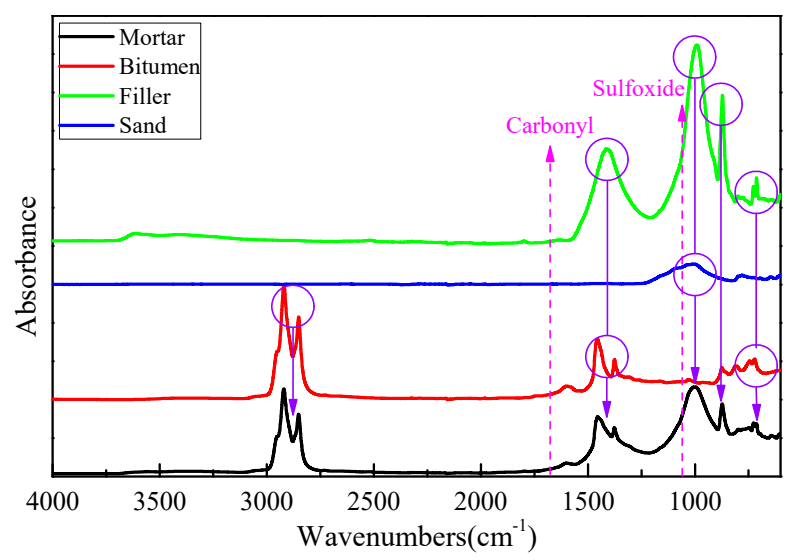

Figure 4. FTIR spectrum of mortar, bitumen, filler, and sand at fresh state. 


\subsubsection{Ageing Index}

In this study, the effects of ageing were analyzed considering specific bands of wavenumber as defined by Lamontagne et al. [28-30] and the corresponding area under those bands. Then, the carbonyl and the sulfoxide indices were determined by dividing the area under a specific location of the spectrum by the sum of specific areas, Equations (1) and (2).

$$
\begin{aligned}
& \text { Carbonyl index }=\frac{A_{1700}}{\sum A} \\
& \text { Sulfoxide index }=\frac{A_{1030}}{\sum A}
\end{aligned}
$$

where $\sum A=A_{(2953,2862)}+A_{1700}+A_{1600}+A_{1460}+A_{1376}+A_{1030}+A_{864}+A_{814}+A_{743}+A_{724}$.

Using the obtained spectra, the ageing indices (carbonyl and sulfoxide index) of each sample were calculated. Figure 5 shows the carbonyl and sulfoxide indices (average value of three measurements) of bitumen, mortar_AM, and mortar_MA. The error bar in Figure 5 is the standard deviation of the three measurements. It can be observed that under the same ageing conditions, the value of the carbonyl index for mortar_AM was similar to that of bitumen. This can be attributed to the fact that carbonyls in the mortar only came from bitumen as illustrated in Figure 4. Previous studies have shown that carbonyls are formed later than sulfoxides because sulfur is more reactive than carbon in bitumen. Later, carbonyls increases due to the full consumption of sulfur [31]. Similar trends and values were found for the mortar in our study as well.

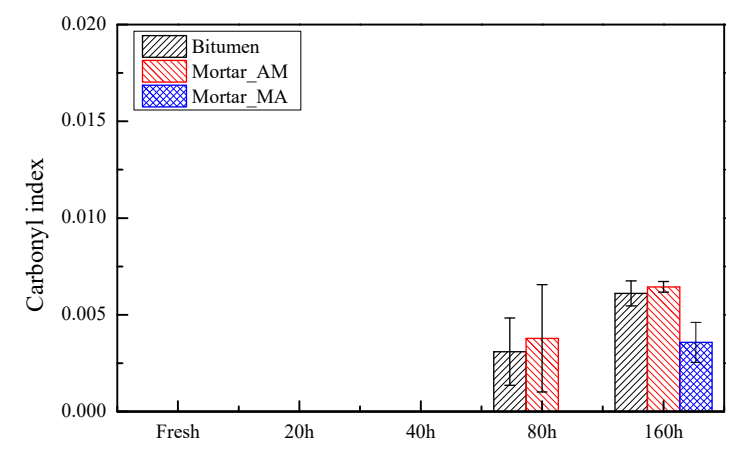

(a)

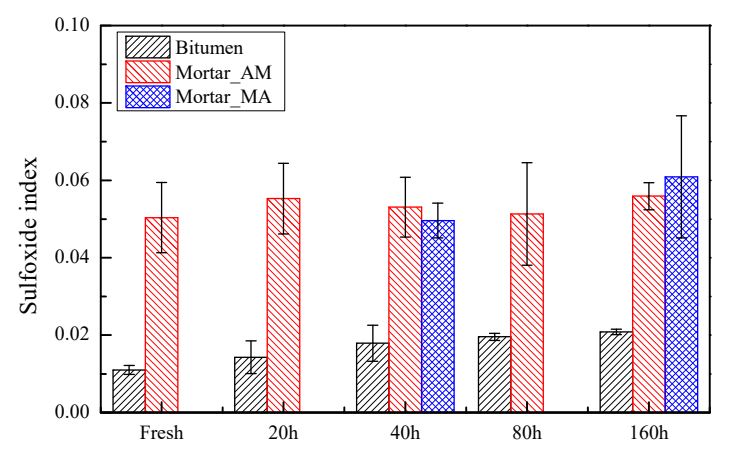

(b)

Figure 5. Function group index of mortar at different ageing conditions: (a) carbonyl index; (b) sulfoxide index.

Moreover, it can be observed that the carbonyl index of mortar_MA was significantly smaller than that of mortar_AM (Figure 5a), indicating that mortar_MA had a lower ageing rate. This finding confirms the theory that the sand and filler particles present in the mortar can slow down the diffusion rate of oxygen due to an increased length of diffusion paths and consequently slow down the ageing process as well [32]. In addition, the diffusivity of mortar will decrease as the bitumen volume fraction decreases [33]. This happens because mortar is the mixtures of bitumen, filler, and sand, and its diffusivity should always be between that of the bitumen and the filler/sand. Moreover, the diffusivity of bitumen is much higher than that of filler/sand $[34,35]$.

The sulfoxide index of mortar_AM differed significantly with that of bitumen (Figure $4 b$ ). This was because sulfoxides in the mortar came not only from the bitumen component but also from the filler and sand particles as illustrated in Figure 3. The sulfoxides content in filler and sand was much higher than that in bitumen, and this led to obvious change in the sulfoxide index of the mortar. Moreover, as another result, the deviation of the mortar results was obviously higher than that of bitumen. The reason for this might be because the measuring area of the FTIR test was limited, meaning the 
infrared radiation hit only one or few of the components of mortar. Another reason might be that the mix of mortar samples was not homogenous.

\subsection{Dynamic Shear Rheometer}

\subsubsection{Frequency Sweep Tests}

At least three replicate samples were tested in DSR at each condition. Based on the time-temperature superposition (TTS) principle, master curves of complex shear modulus and phase angle were generated at reference temperature of $20^{\circ} \mathrm{C}$. Figure 6 shows the evolution of the rheological characteristics of bitumen with increased ageing time. The master curves of mortar_AM and mortar_MA at various ageing times are shown in Figure 7.

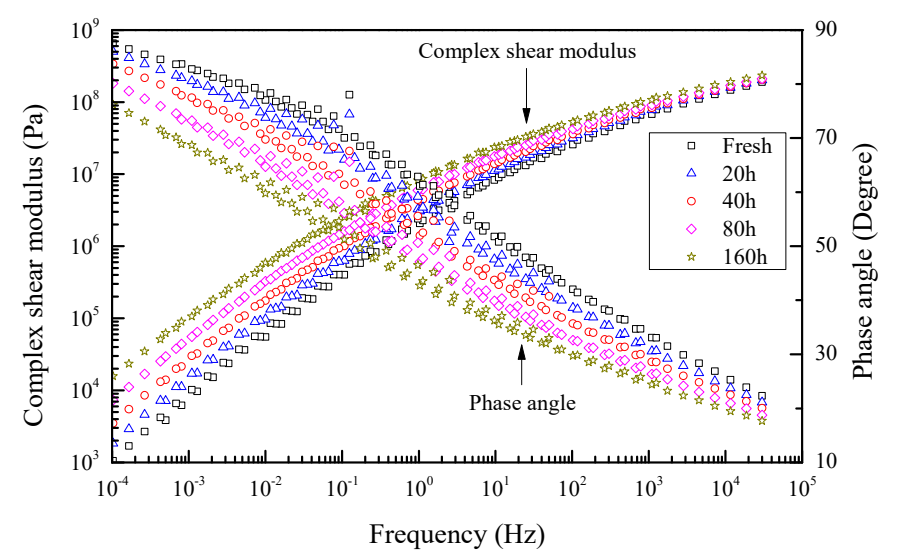

Figure 6. Master curves of PEN 70/100 at different ageing times.

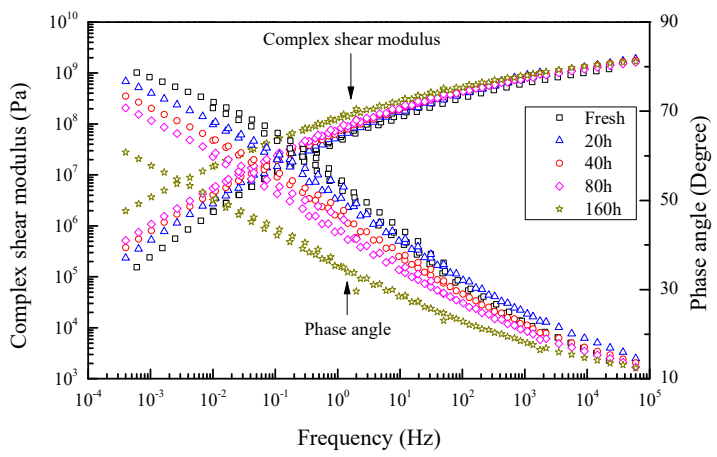

(a)

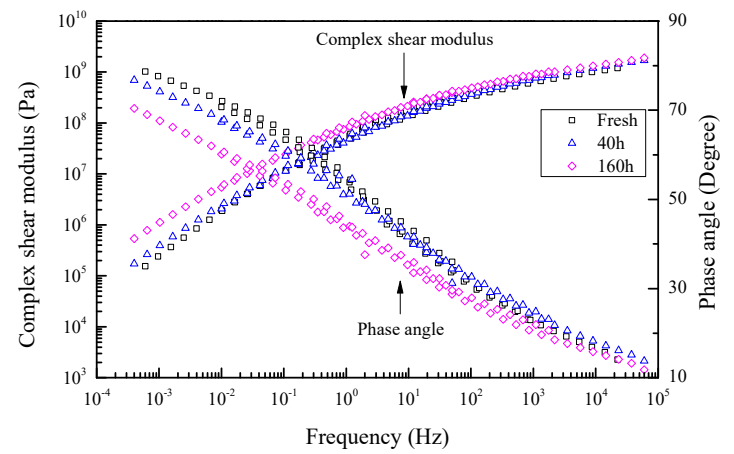

(b)

Figure 7. Master curve of mortar samples at different ageing times: (a) mortar_AM; (b) mortar_MA.

As shown in Figure 6, the observed differences were more pronounced at low frequencies, while they were diluted at higher frequencies. Specifically, the variation of complex modulus between fresh and aged materials was about one order of magnitude at low frequencies. Moreover, at the highest frequency, all samples tended to reach an asymptote at a value of $10^{8} \mathrm{~Pa}$. On the contrary, the phase angle differed substantially for the whole frequency range. The greater the value of complex shear modulus, the harder was the bitumen; the smaller the phase angle, the more elastic was the bitumen.

In Figure 7, it can be seen that the complex shear modulus of mortar sample increased as expected with increasing ageing, while the opposite was observed for the phase angle. Specifically, the variation of complex modulus between fresh and aged materials was one order of magnitude at low frequencies. Moreover, at the highest frequency, all samples tended to reach an asymptote at a value of $10^{9} \mathrm{~Pa}$. The threshold value at high frequency was one order of magnitude larger than that of bitumen due to the addition of filler and sand. On the other hand, the phase angle of the mortar samples differed 
substantially for the whole frequency range. These results show that the material became stiffer and more brittle with ageing. The comparison between mortar_AM and mortar_MA is shown in Figure 8.

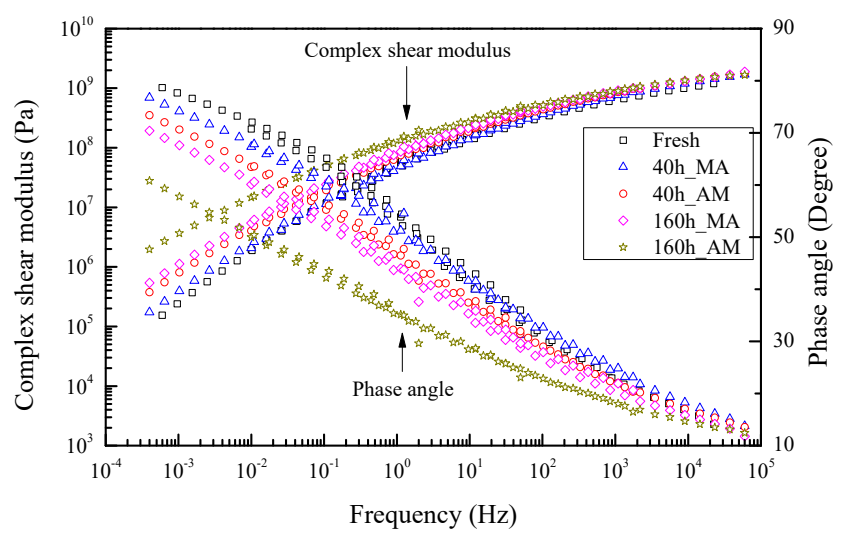

Figure 8. Master curve of mortar_AM and mortar_MA at different ageing times.

Figure 8 shows that compared to mortar_AM, mortar_MA had lower values of complex shear modulus and higher values of phase angle. In other words, mortar_MA was softer under the same ageing conditions, thus the effect of ageing was less. This finding was in agreement with the FTIR results presented in Figure 5. Figure 9 shows the differences in the complex shear modulus and phase angle at $20^{\circ} \mathrm{C}$ and at $10 \mathrm{~Hz}$ frequency for the bitumen, mortar_MA, and mortar_AM samples. The reason for choosing shear moduli at $10 \mathrm{~Hz}$ and $20{ }^{\circ} \mathrm{C}$ was that $10 \mathrm{~Hz}$ is the commonly used frequency in a dynamic test and $20^{\circ} \mathrm{C}$ is considered to be room temperature [36-38].

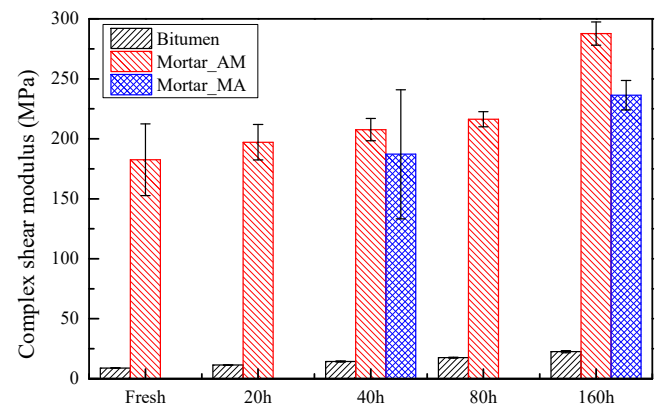

(a)

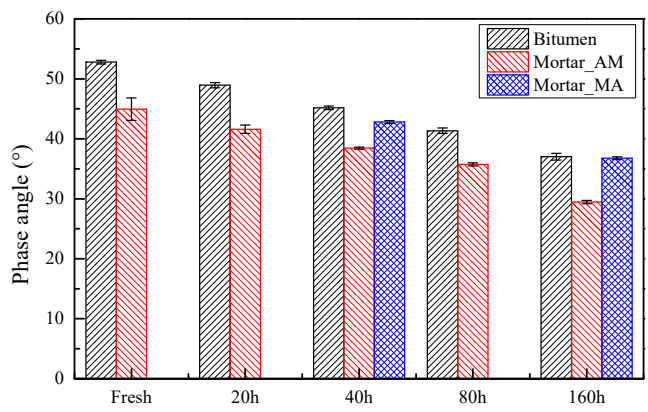

(b)

Figure 9. Complex shear modulus and phase angle at $20^{\circ} \mathrm{C}, 10 \mathrm{~Hz}$ of bitumen, mortar_AM, and mortar_MA.: (a) complex shear modulus; (b) phase angle.

It can be observed that the complex shear modulus of mortar was significantly higher than that of bitumen, with the variation being about 200 times higher, while the phase angle of mortar was slightly smaller than that of bitumen. This high difference in stiffness occurred due to the presence of filler and sand particles, while the phase angle of mortar was mainly determined by the bitumen component.

\subsubsection{Uniaxial Tension Tests}

Uniaxial tension tests were performed using DSR. To obtain the effect of ageing on the tensile behavior of asphalt mortar, at least three samples were tested for each ageing condition. Tensile strength and failure strain are influenced by varying the temperature and strain rate due to the time-temperature dependency of bituminous materials. The tensile strength is higher for lower temperatures and higher strain rates. Therefore, uniaxial tensile tests in this study were performed at $0{ }^{\circ} \mathrm{C}$ with $0.2 \%(0.024 \mathrm{~mm} / \mathrm{s})$ tensile rate to make sure all tests can be conducted in a suitable testing range of the device. The normal force in relation to the displacement of the machine for mortar_AM at different ageing times was recorded and is plotted in Figure 10. 


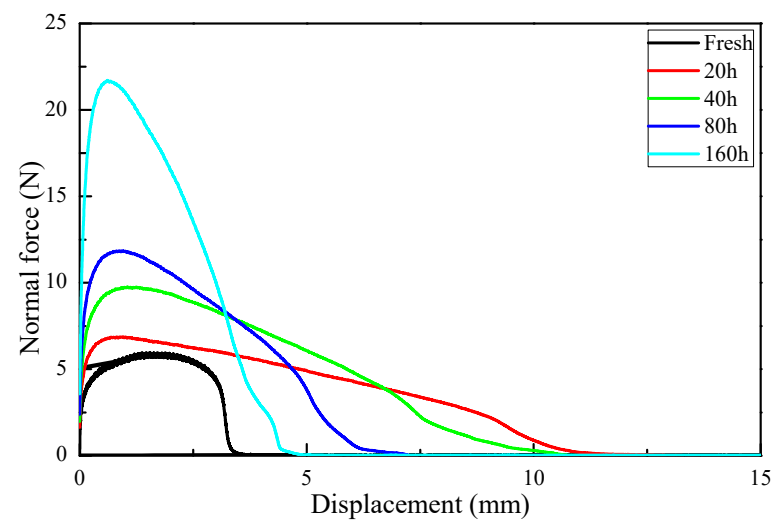

Figure 10. Normal force vs. displacement of mortar_AM with different ageing times.

Figure 10 shows that higher peak normal forces and smaller displacement at failure were obtained for longer ageing times. In other words, aged samples tended to become more brittle. Meanwhile, the steeper initial slopes of the curves can be seen at the longer ageing times, which mean higher moduli for the aged sample. Tensile strength is the maximum normal force divided by the crossing area of the cylinder sample. It denotes the maximum stress that a material can withstand while being pulled before breaking. Figure 11 shows the tensile strength of asphalt mortar at different ageing times. It can be observed that the strength of mortar increased with ageing time. Another observation was that under the same ageing conditions, the strength of mortar_AM was higher than that of mortar_MA.

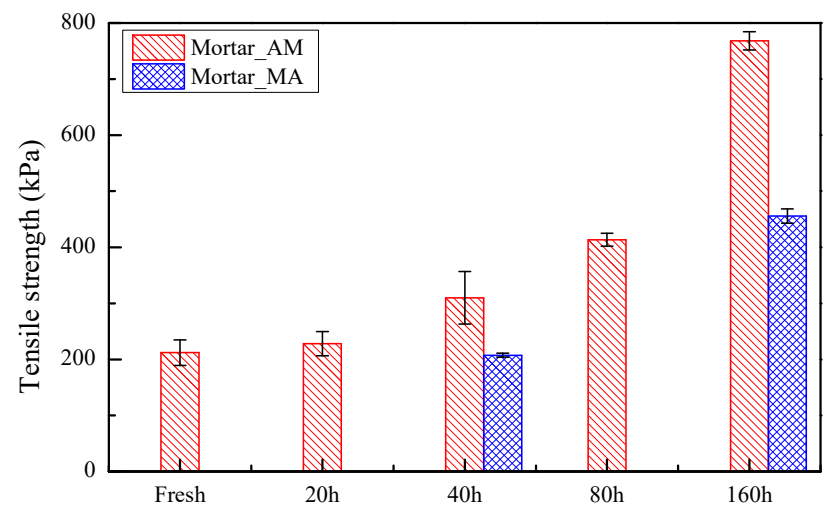

Figure 11. Tensile strength of mortar_AM and mortar_MA with different ageing times.

\section{Conclusions}

Based on the findings and analysis presented in this paper, the following conclusions can be drawn: (1) Asphalt mortar — a mixture of bitumen, filler, and sand-has all functional groups as its three component materials. Carbonyl group that is present in the mortar originates only from the presence of bitumen, whereas sulfoxides can be present in all three components. (2) Ageing increases the complex shear modulus and decreases the phase angle of bitumen and asphalt mortar. The high difference in stiffness observed between bitumen and asphalt mortar is due to the presence of filler and sand particles. The less difference observed between the phase angle values of bitumen and mortar is due to the fact that the phase angle is mainly governed by the bitumen component. (3) Ageing leads to higher strength and smaller displacement at failure compared to unaged sample. In other words, aged samples tend to become more brittle. (4) Both chemical and mechanical test results have shown that the sand and filler particles present in the mortar may slow down the diffusion rate of oxygen. The hypothesis is that the presence of particles increases the length of the diffusion path and therefore slows down the ageing process. This will help to understand the interaction between components during ageing and develop a more suitable protocol to simulate ageing procedure of bituminous materials in the field. 
The results of this study will help to understand the ageing process of asphalt mortar and more accurately predict evolution of its properties due to ageing. Based on the results, the relationship between the chemical and mechanical properties of asphalt mortar can be determined, and a chemo-mechanical model of asphalt mortar can be developed and implemented using the finite element method.

Author Contributions: R.J., X.L., and A.V. conceived the experimental plan and contributed to the interpretation of the results. R.J. carried out the experiments. A.S. and S.E. supervised all project activities.

Funding: This research received no external funding.

Acknowledgments: The authors gratefully acknowledge the Dutch Ministry of Transport, Public Works, and Water Management for funding this project.

Conflicts of Interest: The authors declare no conflicts of interest.

\section{References}

1. Molenaar, A.A.; Meerkerk, A.J.; Miradi, M.; Van der Steen, T. Performance of porous asphalt concrete. J. Assoc. Asphalt Paving Technol. 2006, 75, 1053-1094.

2. Hagos, E.T. The Effect of Aging on Binder Properties of Porous Asphalt Concrete. Ph.D. Thesis, Delft University of Technology, Delft, The Netherlands, 2008.

3. Huber, G. Performance Survey on Open-Graded Friction Course Mixes; Transportation Research Board: Washington, DC, USA, 2000; Volume 284.

4. Yildirim, Y.; Dossey, T.; Fults, K.W.; Trevino, M. Winter Maintenance Issues Associated with New Generation Open-Graded Friction Courses; Center for Transportation Research, The University of Texas at Austin: Austin, TX, USA, 2006.

5. Huurman, M.; Mo, L.-T.; Woldekidan, M.F. Porous asphalt ravelling in cold weather conditions. Int. J. Pavement Res. Technol. 2010, 3, 110-118.

6. Airey, G.D.; Choi, Y.K. State of the Art Report on Moisture Sensitivity Test Methods for Bituminous Pavement Mater. Road Mater. Pavement Des. 2002, 3, 355-372. [CrossRef]

7. Saoula, S.; Soudani, K.; Haddadi, S.; Munoz, M.E.; Santamaria, A. Analysis of the rheological behavior of aging bitumen and predicting the risk of permanent deformation of asphalt. Mater. Sci. Appl. 2013, 4, 312-318. [CrossRef]

8. Siddiqui, M.N.; Ali, M.F. Investigation of chemical transformations by NMR and GPC during the laboratory aging of Arabian asphalt. Fuel 1999, 78, 1407-1416. [CrossRef]

9. Lv, S.; Wang, S.; Guo, T.; Xia, C.; Li, J.; Hou, H. Laboratory evaluation on performance of compound-modified asphalt for rock asphalt/styrene-butadiene rubber (SBR) and rock asphalt/nano-caco 3 . Appl. Sci. 2018, 8, 1009. [CrossRef]

10. Hardesty, J.H.; Attili, B. Spectrophotometry and the Beer-Lambert Law: An Important Analytical Technique in Chemistry; Collin College: Collin, TX, USA, 2010.

11. Huurman, M.; Mo, L.; Woldekidan, M.F. Unravelling porous asphalt concrete towards a mechanistic materials design tool. Road Mater. Pavement Des. 2010, 11, 583-612. [CrossRef]

12. He, Y.; Mohammad, Z.A.; Jones, D.; Harvey, J. Proposing a solvent-free approach to evaluate the properties of blended in asphalt mixes containing high quantities of reclaimed asphalt pavement and recycled asphalt shingles. Construct. Build. Mater. 2016, 114, 172-180. [CrossRef]

13. Kliewer, J.E.; Bell, C.A.; Sosnovske, D.A. Investigation of the Relationship between Field Performance and Laboratory Aging Properties of Asphalt Mixtures, in Engineering Properties of Asphalt Mixtures and the Relationship to Their Performance; ASTM International: West Conshohocken PA, USA, 1995.

14. Cui, S.; Deng, H.; Mo, L. Effect of laboratory long-term ageing simulation testing on response properties of bitumen and bituminous mortar. World Build. Mater. 2012, 33, 35-38.

15. Molenaar, A.A.A.; Hagos, E.T.; Van de Ven, M.F.C. Effects of aging on the mechanical characteristics of bituminous binders in PAC. J. Mater. Civ. Eng. 2010, 22, 779-787. [CrossRef]

16. Mo, L.; Huurman, M.; Wu, S.; Molenaar, A.A.A. Ravelling investigation of porous asphalt concrete based on fatigue characteristics of bitumen-stone adhesion and mortar. Mater. Des. 2009, 30, 170-179. [CrossRef] 
17. Tong, Y.; Luo, R.; Lytton, R.L. Moisture and ageing damage evaluation of asphalt mixtures using the repeated direct tensional test method. Int. J. Pavement Eng. 2015, 16, 397-410. [CrossRef]

18. Zhang, Y.; Leng, Z. Quantification of bituminous mortar ageing and its application in raveling evaluation of porous asphalt wearing courses. Mater. Des. 2017, 119, 1-11. [CrossRef]

19. Huurman, M. Lifetime Optimisation Tool-Main Report; Report 7-07-170-1; Delft University of Technology: Delft, The Netherlands, 2007.

20. Mo, L. Damage Development in the Adhesive Zone and Mortar of Porous Asphalt Concrete. Ph.D. Thesis, Delft University of Technology, Delft, The Netherlands, 2010.

21. Van den Bergh, W. The Effect of Ageing on the Fatigue and Healing Properties of Bituminous Mortars. Ph.D. Thesis, Delft University of Technology, Delft, The Netherlands, 2011.

22. Mohammad, J.K.; Kyatham, V. Viscoelastic behavior of hydrated lime-modified asphalt matrix and hot-mix asphalt under moisture damage conditions. J. Transp. Res. Board 2008, 2370, 64-74.

23. Kuang, D.; Yu, J.; Feng, Z.; Li, R.; Chen, H.; Guan, Y.; Zhang, Z. Performance evaluation and preventive measures for aging of different bitumen. Construct. Build. Mater. 2014, 66, 209-213. [CrossRef]

24. Ahmed, F. Diffusion of the Rejuvenators into Bitumen Studied by FTIR-ATR as a Function of Temperature and Bitumen Properties; Aalto University: Helsinki, Finland, 2016.

25. Kandhal, P.; Chakraborty, S. Effect of asphalt film thickness on short-and long-term aging of asphalt paving mixtures. Transp. Res. Record 1996, 1535, 83-90. [CrossRef]

26. Ruan, Y.; Davison, R.R.; Glover, C.J. An Investigation of Asphalt Durability: Relationships Between Ductility and Rheological Properties for Unmodified Asphalts. Petrol. Sci. Technol. 2003, 21, 231-254. [CrossRef]

27. Lesueur, D. The colloidal structure of bitumen: Consequences on the rheology and on the mechanisms of bitumen modification. Adv. Colloid Interface Sci. 2009, 145, 42-82. [CrossRef] [PubMed]

28. Lamontagne, J.; Dumas, P.; Mouillet, V.; Kister, J. Comparion by Fourier Transform Infrared (FTIR) Spectrocopy of Different Ageing Techniques: Application to Road Bitumen. Fuel 2001, 80, 483-488. [CrossRef]

29. Hofko, B.; Alavi, M.Z.; Grothe, H.; Jones, D.; Harvey, J. Repeatability and sensitivity of FTIR-ATR spectral analysis method for bituminous binders. Mater. Struct. 2017, 50, 187. [CrossRef]

30. Hofko, B.; Porot, L.; Cannone, A.F.; Poulikakos, L.; Huber, L.; Lu, X.; Mollenhauer, K.; Grothe, H. FTIR spectral analysis of bituminous binders: Reproducibility and impact of ageing temperature. Mater. Struct. 2018, 51, 45. [CrossRef]

31. Jing, R.; Varveri, A.; Liu, X.; Scarpas, A.; Erkens, S. Chemo-mechanics of ageing on bitumen materials. In Proceedings of the Transportation Research Board 97th Annual Meeting Transportation Research Board, Washington, DC, USA, 7-11 January 2018.

32. Weller, K.R.; Stenhouse, N.S.; Watts, H. Diffusion of Gases in Porous Solids. I. Theoretical Background and Experimental Method. Can. J. Chem. 1974, 52, 2684-2691. [CrossRef]

33. Das, P.K.; Balieu, R.; Kringos, N.; Birgisson, B. On the oxidative ageing mechanism and its effect on asphalt mixtures morphology. Mater. Struct. 2015, 48, 3113-3127. [CrossRef]

34. Jing, R.; van Lent, D.; Kasbergen, C.; Scarpas, A.; Liu, X.; Erkens, S. Experimental and computational investigation of gas diffusion in bitumen. In Proceedings of the Transportation Research Board 96th Annual Meeting Transportation Research Board, Washington, DC, USA, 8-12 January 2017.

35. Peng, S.; Hu, Q.; Hamamoto, S. Diffusivity of rocks: Gas diffusion Measurements and correlation to porosity and pore size distribution. Water Resour. Res. 2012, 48, W02507. [CrossRef]

36. Rossi, C.O.; Caputo, P.; Loise, V.; Miriello, D.; Teltayev, B.; Angelico, R. Role of a food grade additive in the high temperature performance of modified bitumens. Colloids Surf. A Physicochem. Eng. Aspects 2017, 532, 618-624. [CrossRef]

37. Filonzi, A.; Delgadillo, R. Sample size correction factors for indentation on asphalt bitumens. Constr. Build. Mater. 2017, 154, 877-883. [CrossRef]

38. Schlosser, F.; Mikolaj, J.; Zatkalikova, V.; Sramek, J.; Durekova, D.; Remek, L. Deformation properties and fatigue of bituminous mixtures. Adv. Mater. Sci. Eng. 2013, 2013, 701764. [CrossRef]

(C) 2018 by the authors. Licensee MDPI, Basel, Switzerland. This article is an open access article distributed under the terms and conditions of the Creative Commons Attribution (CC BY) license (http:/ / creativecommons.org/licenses/by/4.0/). 